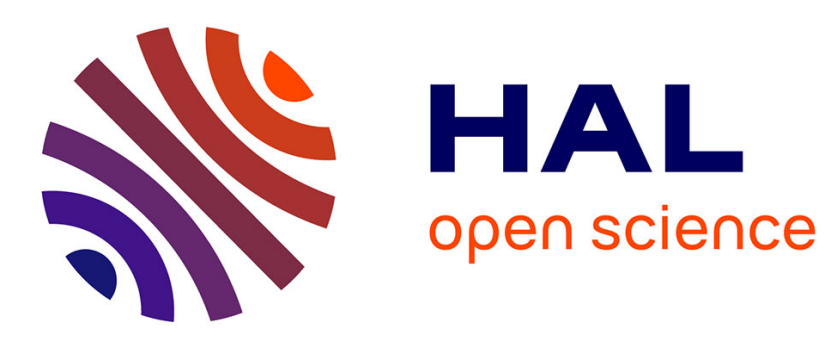

\title{
Génération d'ondes acoustiques dans un milieu anisotrope par impact laser
}

\author{
A. Mourad, M. Deschamps, Bernard Castagnede
}

\section{To cite this version:}

A. Mourad, M. Deschamps, Bernard Castagnede. Génération d'ondes acoustiques dans un milieu anisotrope par impact laser. Journal de Physique IV Proceedings, 1994, 04 (C5), pp.C5-749-C5-752. 10.1051/jp4:19945162 . jpa-00252842

\section{HAL Id: jpa-00252842 https://hal.science/jpa-00252842}

Submitted on 1 Jan 1994

HAL is a multi-disciplinary open access archive for the deposit and dissemination of scientific research documents, whether they are published or not. The documents may come from teaching and research institutions in France or abroad, or from public or private research centers.
L'archive ouverte pluridisciplinaire HAL, est destinée au dépôt et à la diffusion de documents scientifiques de niveau recherche, publiés ou non, émanant des établissements d'enseignement et de recherche français ou étrangers, des laboratoires publics ou privés. 


\title{
Génération d'ondes acoustiques dans un milieu anisotrope par impact laser
}

\author{
A. MOURAD, M. DESCHAMPS et B. CASTAGNÈDE*
}

\author{
Laboratoire de Mécanique Physique, Université Bordeaux I, 33405 Talence, France \\ * Laboratoire d'Acoustique de l'Université du Maine, Av. Olivier Messiaen, BP. 535, 72017 Le Mans cedex, \\ France
}

The Lamb's problem for an elastic, anisotropic and homogeneous half space is solved. The source is linear and contains in the free surface of this half space. A numerical calculation based on the Cagniard de Hoop method is performed, in the case of cubic symmetry. The free surface is parallel to a plane of symmetry. In addition, the line source may be parallel, or not, to an axis of symmetry. The displacement field is then calculated at any point of this half space, for various directions of this line source. This theoretical study may be applied to the generation of acoustic waves when using a laser beam focused by a cylindrical lens on a sample. The impact is then linear.

\section{1 - Introduction.}

E. A. Kraut $\left({ }^{1}\right)$ est le premier qui a résolu le problème de Lamb pour un milieu anisotrope par la méthode de Cagniard-de Hoop, pour une source linéaire confondue avec un axe de symétrie (deux modes sont alors générés). Par la suite, des résultats numériques ont été effectué pour un récepteur placé à l'épicentre ou à la surface (2). En dehors de ces deux cas de figure, il n'existe pas de résultats numériques précis, notamment lorsque la source est sécante à un axe de symétrie (trois modes sont générés). C'est de ce dernier cas dont il question dans le présent travail.

Récemment Every et Kim ont résolu le même problème pour un impact ponctuel et obtenu la fonction de Green correspondante à un échelon par la méthode de la transformée de Radon ( ${ }^{3}$ ). Ce qui modélise la cassure d'un capillaire à la surface d'un échantillon $\left(^{4}\right)$. Il est alors nécessaire d'effectuer un quadrillage de pas moins de quatre millions de points dans l'espace des fréquences spatiales, pour obtenir une solution convenable.

Deux intérêts se dégagent de notre étude. D'une part, d'un point de vue théorique, la fonction de Green pour une ligne source est rapide à obtenir (aucune intégration n'est à faire). D'autre part, d'un point de vue expérimental, une ligne source peut être réalisée en génération d'ondes acoustiques par impact laser, en focalisant le faisceau laser avec une lentille cylindrique. De plus, le calcul de la fonction de Green pour une source ponctuelle ne nécessitera alors qu'une seule intégration, en considérant un faisceau de lignes sources, et donnera vraisemblablement de meilleurs résultats que l'emploie de la transformée de Radon.

\section{2 - Fonction de Green.}

Supposons une source linéaire localisée sur la surface d'un demi-espace élastique (fig. 1-a). Le repère fixe $\left(\underline{\mathbf{X}}_{1}, \underline{\mathbf{X}}_{2}, \underline{\mathbf{X}}_{3}\right)$ est confondu avec les axes de symétrie du milieu orthotrope. La ligne est dirigée suivant l'axe $\underline{\mathbf{X}}^{\prime} 2=(0, \cos \phi, \sin \phi)$. Le problème est alors plan et invariant par translation suivant l'axe $\underline{\mathbf{X}}_{2}^{\prime}$. De ce fait, nous travaillions dans le repère lié à la source $\left(\underline{\mathbf{X}}_{1}, \underline{\mathbf{X}}^{\prime}{ }^{3}\right)$. La composante normale du 
champ de déplacement (suivant $\underline{\mathbf{X}}_{1}$ ) est calculée en tout point $M\left(\mathrm{x}_{1}, \mathrm{x}^{\prime}{ }_{3}\right)=\mathrm{M}(\mathrm{r}, \theta)$, cf.(fig. 1-b), en fonction du temps. D'autre part, nous astreignons le point $M$ à se déplacer dans le plan parallele à la surface libre situé à une distance fixe e de celle-ci.

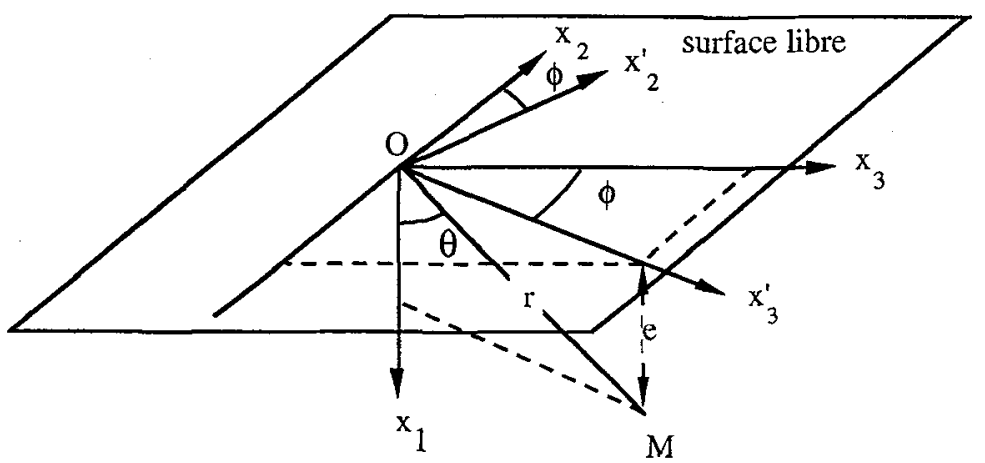

fig. 1-a

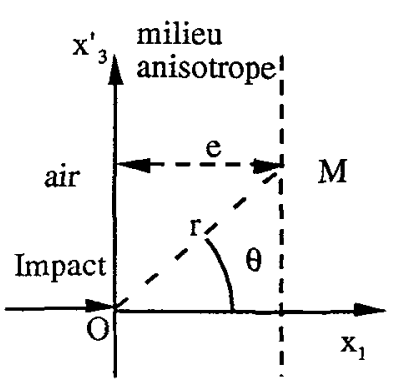

fig. 1-b

Nous proposons de mettre en équation le problème. Si on écrit les composantes du champs de déplacement sous la forme suivante dans le repère fixe:

$$
\mathrm{U}\left(\mathrm{u}_{1}, \mathrm{u}_{2}, \mathrm{u}_{3}\right)=\left(\mathrm{u}_{1}\left(\mathrm{x}_{1}, \mathrm{x}_{2}, \mathrm{x}_{3}\right), \mathrm{u}_{2}\left(\mathrm{x}_{1}, \mathrm{x}_{2}, \mathrm{x}_{3}\right), \mathrm{u}_{3}\left(\mathrm{x}_{1}, \mathrm{x}_{2}, \mathrm{x}_{3}\right)\right) \text {. }
$$

Il doit vérifier le système d'équations aux dérivées partielles suivant:

- équation de propagation ( $c_{\mathrm{ijkl}}$ est le tenseur d'élasticité rapporté à la masse volumique),

- conditions limites,

$$
c_{i j k i} \frac{\partial u_{k}}{\partial x_{j} \partial x_{1}}=\frac{\partial^{2} u_{i}}{\partial t^{2}}
$$

$$
\mathrm{c}_{\mathrm{i} 1 \mathrm{kl}} \frac{\partial \mathrm{u}_{\mathrm{k}}}{\partial \mathrm{x}_{1}}=-\mathrm{s}_{\mathrm{i} 1} \delta\left(-\mathrm{x}_{2} \sin \phi+\mathrm{x}_{3} \cos \phi\right) \delta(\mathrm{t}), \text { pour } \mathrm{x}_{1}=0 \text { et } \forall\left(\mathrm{x}_{2}, \mathrm{x}_{3}, \mathrm{t}\right)
$$

- conditions de Sommerfeld,

$$
\lim _{x_{1} \rightarrow \infty}\left(u_{i}, \frac{\partial u_{i}}{\partial x_{k}}, \frac{\partial^{2} u_{i}}{\partial x_{k}^{2}}, \frac{\partial u_{i}}{\partial t}, \frac{\partial^{2} u_{i}}{\partial t^{2}}\right)=0, \forall(i, k) \in(1,2,3) \times(1,2,3),
$$

- causalité,

$$
\left(\frac{\partial \mathrm{U}}{\partial \mathrm{t}}, \mathrm{U}\right)=(0,0) \text { pour } \mathrm{t}<0
$$

Ces conditions limites modélisent la configuration expérimentale de la génération d'ondes acoustiques par impact laser en focalisation cylindrique, en régime d'ablation $(5)$. On trouvera plus de précisions sur les applications du laser à l'acoustique dans l'article de Castagnède B. et Berthelot Y. $\left({ }^{6}\right)$. Le système précédent est résolu par la méthode de la double transformées mixte de Fourier (symbolisée par une tilde) sur l'espace et de Laplace (symbolisée par une barre) sur le temps. L'original de la solution est recherché par la méthode de Cagniard- de Hoop. L'image de la solution s'écrit:

$$
\bar{u}_{1}(r, \theta, s)=\frac{1}{2 \pi i} \sum_{j=1}^{3} \int_{-i \infty}^{+i \infty} \overline{\tilde{f}}_{j}(p) \exp -s r\left(p \sin \theta+\eta_{j}(p) \cos \theta\right) d p,
$$

avec: s: le paramètre de la transformée de Laplace, p: la composante de la lenteur de phase suivant $x_{3}$, $\eta_{\mathrm{j}}$ : la composante de la lenteur de phase suivant $\mathrm{x} 1$, de chacun des trois modes. 
Le changement de variable de Cagniard: $t=r\left(p \sin \theta+\eta_{j}(p) \cos \theta\right)$, donne l'original :

$$
\mathrm{u}_{1}(\mathrm{r}, \theta, \mathrm{t})=\frac{1}{\pi} \sum_{j=1}^{3} \operatorname{Im}\left[\overline{\tilde{f}}_{j}\left(\mathrm{p}_{\mathrm{j}}(\mathrm{t})\right) \frac{d \mathrm{p}_{\mathrm{j}}}{d t}\right] \text {, c'est la fonction de Green du problème. }
$$

Pour plus de détails sur la méthode, on pourra se référer aux travaux de Van der Hijden $\left({ }^{7}\right)$.

\section{3) Résultats numériques pour une symétrie cubique.}

Les caractéristiques de l'échantillon sont les suivantes:

$\mathrm{C}_{11}=\mathrm{C}_{22}=\mathrm{C}_{33}=11.88 \mathrm{GPa}$;

$\mathrm{C}_{12}=\mathrm{C}_{13}=\mathrm{C}_{23}=5.38 \mathrm{GPa}$;

$\mathrm{C}_{44}=\mathrm{C}_{55}=\mathrm{C}_{66}=7.94 \mathrm{GPa}$;

$\mathrm{e}=5 \mathrm{~mm}$,

densité $=1.5$.

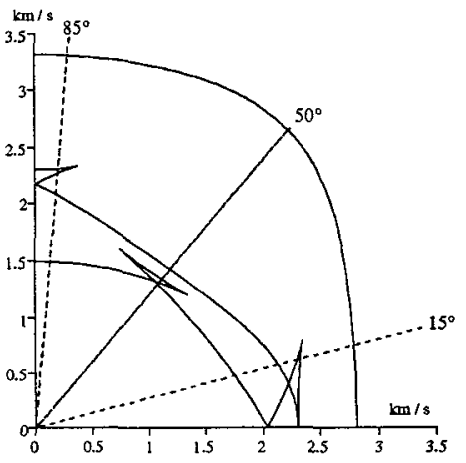

fig. 3. Courbes de vitesses de groupe $\left(\phi=40^{\circ}\right)$.
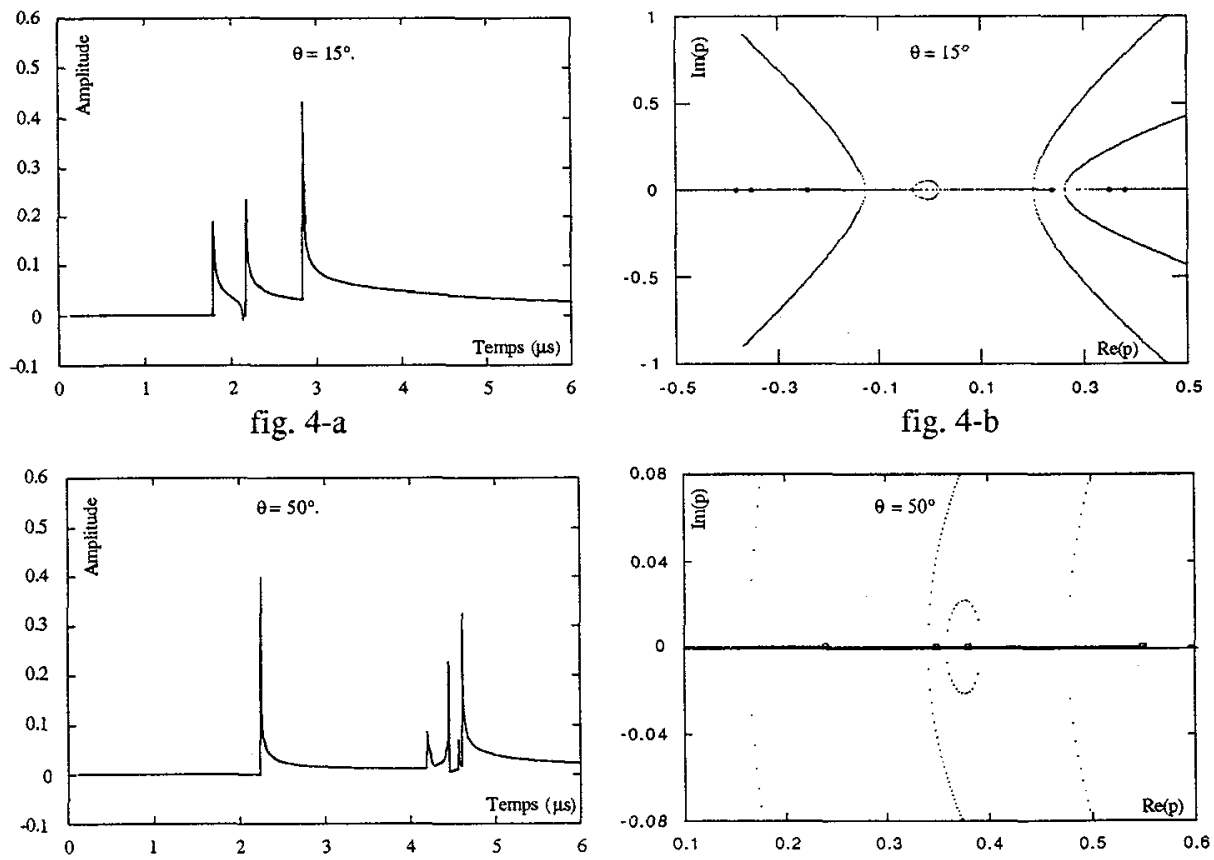

fig. $5-a$

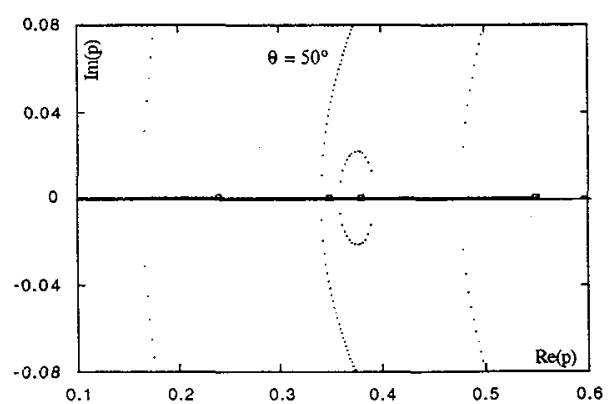

fig. 5-b 


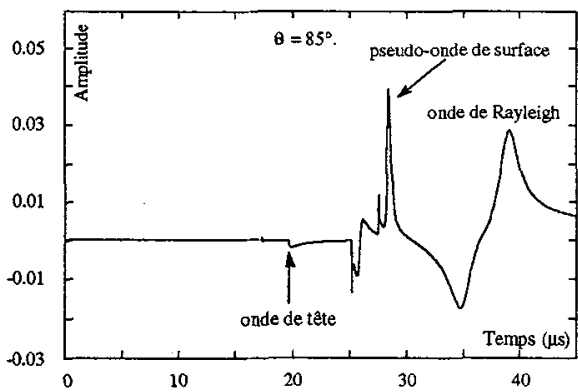

fig. 6-a

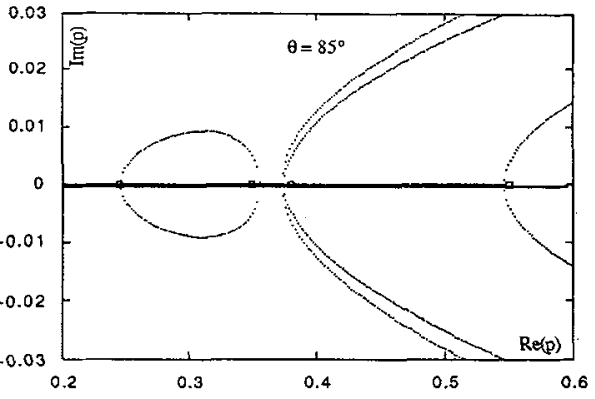

fig. 6-b

Les figures ci-dessus représentent les champs de déplacement (fig. 4 a - 5 a - 6 a) et leurs contours de Cagniard - de Hoop associés (fig. $4 \mathrm{~b}-5 \mathrm{~b}-6 \mathrm{~b}$ ), pour trois directions $\left(15^{\circ}, 50^{\circ}, 85^{\circ}\right)$ indiquées sur les courbes de vitesse de groupe (fig. 3 ). Les contours sont paramètrés par le temps. Les instants où les contours quittent, ou reviennent, sur l'axe réel (points doubles), correspondent à l'arrivée d'une onde. Il a été montré expérimentalement que ces temps correspondent à des mesures de vitesses de groupe $\left({ }^{8}\right)$. Notamment pour $\theta=50^{\circ}$, la droite issue de l'origine intercepte cinq fois les courbes de vitesse de groupe ( fig. 3). En effet les contours admettent cinq points doubles (fig. 5 - b) et le champ de déplacement cinq discontinuités infinies (fig. 5 - a). Le nombre de ces points supérieur à trois atteste de la présence d'une corne. Le nombre de discontinuités infinies apparentes est dû aux choix du pas d'échantillonnage trop grand sur le temps. Ceci est remarquable à $15^{\circ}$, où il existe cinq points doubles sur les contours, alors que l'on observe uniquement trois points de discontinuité infinie sur le champ correspondant.

La discontinuité d'ordre un, que l'on remarque aisément après le mode longitudinal, aux environs de $20 \mu \mathrm{s}$. pour $\theta=85^{\circ}$ est l'onde de diffraction (ou onde de tête). A $85^{\circ}$, on remarque l'onde de Rayleigh et une pseudo - onde de surface dont le pic est très élevé.

\section{Conclusion.}

La méthode de Cagniard - de Hoop est élégante et synthétique. Elle est sans conteste la plus adaptée pour résoudre les problème de propagation en régime transitoire. Elle permet de décrire facilement tous les phénomènes physiques par la seule lecture des contours de Cagniard - de Hoop. Dans le présent travail, le tracé judicieux de ces contours, nous a permis, entre autre, de rendre compte, de l'onde de Rayleigh et des effets de la diffraction par la surface libre, sans aucune lourdeur de calculs supplémentaires.

\section{Références.}

[1] Kraut E. A., 1, 1963, pp. $401-448$.

[2] Burridge R., Quart. Journ. Mech. and Applied Math., Vol. XXIV, Pt. 1, 1971., pp. 81 - 98.

[3] Every A.G. et Kim K. Y., à paraître dans le J. A. S. A.

[4] Kim K. Y. et Sachse W., U. F. F. C. symp. 1992.

[5] Mourad A. Castagnède B. et Mottay E., accepté pour publication au J. A. S. M. E.

[6] Castagnède B. et Berthelot Y., J. Acoust. 5, 417 - 453 (1992).

[7] Van der Hijden J. H. M. T., North-Holland series in Applied Math. and Mech., Ed. Achenbach (1987).

[8] Every A.G, Sachse W., Kim K. Y. et Thompson M. O., Phys. Rev. Lett. 65, 1446 - 1449 (1990). 